\title{
Estime De Soi, Soutien Social ET Performance Scolaire Des Élèves Stagiaires Du Lycée Hôtelier De Cocody (Côte D’ivoire)
}

Dr. Essiomle Yawa Ossi ${ }^{*}$
${ }^{1}$ Ecole Normale Supérieure d'Abidjan, Dép
Article History
Received: 04.12 .2021
Accepted: 10.01 .2022
Published: 19.01 .2022
Journal homepage:
https://www.easpublisher.com
Quick Response Code

\begin{abstract}
The scientific literature could suggest that high self-esteem can positively influence students' academic performance. This leads us to ask the following main question: "Does high self-esteem in a student alone promote his academic performance?" This research question allowed us to explore the concept of selfesteem, social support and academic performance. The objective of the study is to analyze the relationship between self-esteem, social support and the academic performance of students. The hypothesis states that high self-esteem, frequency of social support lead to good academic performance. The theoretical grounding of this research is the self-esteem theory of André and Lelord (1999). The study was carried out with student trainees from the Lycée hôtelier de Cocody, with a sample of fifty-five (55) student trainees made up of twenty-nine (29) girls and twenty-six (26) boys. The data collection techniques used are documentary research, the questionnaire and the semi-structured interview. The self-esteem questionnaire according to Duclos, Laporte and Ross (1995) was administered to student interns. The data collected revealed that high self-esteem and the frequency of social support positively influence the academic performance of student interns.

Keywords: Trainee student - Self-esteem - Social support - Academic performance.
\end{abstract} Copyright () 2022 The Author(s): This is an open-access article distributed under the terms of the Creative Commons Attribution 4.0 International License (CC BY-NC 4.0) which permits unrestricted use, distribution, and reproduction in any medium for non-commercial use provided the original author and source are credited.

\section{INTRODUCTION}

L'école moderne est perçue aujourd'hui comme garant de réussite et d'intégration sociale des individus. La communauté éducative (le personnel éducatif, les autorités politiques, les élèves, les parents d'élèves, les partenaires éducatives) est préoccupé par les problèmes d'adaptation scolaire. L'objectif principal de l'école est le développement de compétences et de connaissances chez les apprenants. L'école est un cadre de vie et d'échanges au sein duquel a lieu nombreuses situations de communication et d'interactions entre des personnalités variées avec la communauté éducative, des apprenants, des parents d'élèves. Pour chaque apprenant, elle est un passage obligatoire pour l'atteinte des objectifs éducatifs.

Plusieurs travaux scientifiques ont identifiés les facteurs de la performance scolaire qui sont entre autres les facteurs liés à l'élève, les facteurs environnementaux (Forquin, Bourdieu et Passeron, 1971; Scheerens, 2000; Kouakou et Kablan, 2015). Les premiers travaux sur la performance scolaire ont montré que la réussite scolaire est en lien avec les origines sociales de l'élève (Baudelot et Establet, 1979; Bourdieu et Passeron, 1970), les valeurs intrinsèques aux enseignants et le type d'établissement scolaire fréquenté (Meuret, 2000). Le système éducatif devrait permettre aux élèves d'entrer dans un cycle d'études donné et le terminer normalement sans échec (Scheerens, 2000). Pour Meuret et Morlaix (2006) la réussite scolaire est en lien avec le lieu de scolarisation, des jugements du maittre et de l'origine sociale des enfants. En outre, les enfants qui ont plus de chance de réussir sont ceux qui ont des parents économiquement plus aisés que ceux dont les des parents sont moins favorisés. Duru-Bellat (2003) a montré à travers ses études qu'aussi bien l'origine sociale des élèves que l'environnement scolaire influent sur la performance scolaire. Par ailleurs, Rocheleau (1998) insiste que dans une école performante, le chef d'établissement intègre un style de gestion qui prend en compte l'ensemble des parties prenantes. Dans la littérature scientifique, lorsqu'on évoque le terme de l'estime de soi, on a une multitude de termes utilisés qui sont entre autres : conscience de soi, perception de soi, concept de soi, représentation de soi, image de soi etc. Ces termes ont pour intérêt principal de définir le soi en tant que construction psychique complexe, qui met différemment en valeur certains mécanismes. Dans cette étude, nous allons partir du soi pour définir l'estime de soi. Plusieurs auteurs voient dans le soi, une structure cognitive qui autorise chaque individu à un retour réflexif conscient sur soi-même (Famose, Guerin

*Corresponding Author: Dr. Essiomle Yawa Ossi 
et Sarrazin, 2005). Ceci pour une organisation structurelle des informations à traiter. En général, l'estime de soi est définie comme l'évaluation globale de la valeur de soi en tant qu'individu, le degré de satisfaction de soi-même. Elle est l'un des fondements de l'image de soi. L'élément essentiel de l'estime de soi est la subjectivité qui rend ce concept complexe. Selon Beauregard, Bouffard et Duclos (2000), l'estime de soi est la conscience de la valeur personnelle qu'on se reconnaît qui se manifeste par un ensemble d'attitudes et de croyances qui permettent à l'individu de faire face au monde extérieur et aux difficultés de la vie. Elle est un jugement positif à son propre égard en ce qui concerne ses propres qualités et habiletés. Le regard positif qu'un individu porte sur lui-même est vital pour son équilibre psychologique, car il lui donne le sentiment de sécurité interne nécessaire pour faire face aux difficultés de la vie. Mais un jugement négatif, entraîne des souffrances ayant des conséquences désastreuses sur l'épanouissement d'un individu ainsi que sur l'efficacité de ses actions. L'estime de soi en tant que concept qui participe à la construction du sujet prend une place dans notre conception du bien-être; ceci a fait l'objet de nombreux travaux scientifiques. Pour Pierrehumbert, Zanone, Kauer-Tchicaloff et Plancherel (1988), un haut niveau d'estime de soi ne conduit pas obligatoirement à la réussite scolaire, mais au contraire, un faible niveau d'estime de soi renvoie à l'échec et aux difficultés. Avec l'Inventaire d'Estime de Soi de Coopersmith
(1967) sur des élèves de sixième, Bawa (2007) a montré que les élèves qui ont une estime de soi positive ont un taux de réussite supérieur à ceux qui ont une estime de soi négative. Ces élèves en outre, répondent aux attentes des enseignants, des parents et de leurs pairs. En Côte d'Ivoire, on constate des échecs massifs malgré une amélioration du pourcentage des résultats aux examens de fin d'année. C'est la raison pour laquelle, le système éducatif ivoirien améliore les conditions d'apprentissage grâce aux réformes pédagogiques. Les auteurs comme (Bloom, 1979; Gerardi, 1990) ont montré qu'il existe une relation significative entre le concept de soi et le rendement scolaire. Selon Bariaud \& Bourcet (1998), une estime de soi élevée est associée à des comportements plus adaptés et plus positifs entre autres la confiance en soi, l'anticipation positive de l'avenir, la recherche de soutien social, la confrontation active à la difficulté, etc. En contrario, une estime de soi faible conduit aux attitudes dysfonctionnelles telles que la tristesse, le fatalisme, l'anticipation négative de l'issue de la difficulté, l'évitement, la passivité et le déni. Le concept de l'estime de soi a fait l'objet de plusieurs études durant de nombreuses années. L'estime de soi est un concept polysémique. C'est la raison pour laquelle, il nous paraît judicieux de reprendre à la page suivante, le tableau de Joly (2011) qui nous dresse les différentes approches permettant de caractériser ce qu'est l'estime de soi.

\begin{tabular}{|c|c|c|c|}
\hline Appellations & Dates & Auteurs & $\begin{array}{l}\text { Définitions des différentes appellations de l'estime } \\
\text { de soi }\end{array}$ \\
\hline $\begin{array}{l}\text { Conscience de la valeur de } \\
\text { soi }\end{array}$ & 1890 & James & $\begin{array}{l}\text { L'estime de soi est le rapport entre les aspirations et les } \\
\text { réussites effectives. }\end{array}$ \\
\hline Miroir social & 1902 & Cooley & $\begin{array}{l}\text { L'estime de soi est définie comme une construction } \\
\text { sociale, façonnée par l'ensemble des interactions avec } \\
\text { les autres. }\end{array}$ \\
\hline $\begin{array}{l}\text { Pyramide des besoins de } \\
\text { Maslow }\end{array}$ & 1943 & A. Maslow & $\begin{array}{l}\text { Théorie selon laquelle les motivations d'une personne } \\
\text { résultent de l'insatisfaction de certains de ses besoins. } \\
\text { Ces besoins sont classés par ordre d'importance } \\
\text { besoins physiologiques, de sécurité, sociaux, d'estime } \\
\text { et de réalisation de soi. L'estime de soi résulte du } \\
\text { sentiment d'être utile et d'avoir de la valeur, c'est un } \\
\text { besoin. }\end{array}$ \\
\hline Locus de contrôle & 1966 & Rotter & $\begin{array}{l}\text { Elle est définie comme la croyance qu'un individu est } \\
\text { acteur des événements de sa vie. }\end{array}$ \\
\hline Concept de soi & 1976 & $\begin{array}{l}\text { Shavelson } \\
\text { Hubner\& } \\
\text { Stanton }\end{array}$ & $\begin{array}{l}\text { L'estime de soi n'est pas une réalité psychologique une } \\
\text { et indivisible, c'est un système multidimensionnel et } \\
\text { hiérarchisé, qui correspond à une multitude } \\
\text { d'impressions, de sentiments relatifs à différents } \\
\text { domaines du vécu quotidien. }\end{array}$ \\
\hline Estime de soi & 1978 & L'Ecuyer & $\begin{array}{l}\text { Ensemble de traits, d'images, de sentiments que } \\
\text { l'individu reconnaît comme faisant partie de lui-même } \\
\text { influencé par l'environnement et organisé de façon plus } \\
\text { ou moins consciente. }\end{array}$ \\
\hline Valeur personnelle & 1982 & Harter & $\begin{array}{l}\text { Evaluation qu'un individu fait de sa propre valeur, } \\
\text { c'est-à-dire sa satisfaction en lui même. L'estime de soi } \\
\text { se renforce dans les situations de réussite. Elle exprime } \\
\text { la façon dont chacun s'accepte, se respecte en tant que } \\
\text { personne. }\end{array}$ \\
\hline Autodétermination & 1985 & Deci\&Ryan & $\begin{array}{l}\text { La théorie de l'autodétermination maintient que la } \\
\text { personne tend à satisfaire trois besoins fondamentaux: } \\
\text { le besoin d'autonomie, le besoin de compétence et le } \\
\text { besoin de relation à l'autre. Les situations qui sont à } \\
\text { même de satisfaire ces trois besoins sont celles qui } \\
\text { engendrent le plus de motivation. }\end{array}$ \\
\hline Estime personnelle & 1984 & Coopersmith & $\begin{array}{l}\text { Renvoie à l'évaluation qu'une personne fait d'elle- } \\
\text { même, elle est le reflet d'une attitude d'approbation ou } \\
\text { de désapprobation. }\end{array}$ \\
\hline Valeur de soi & 1995 & Martinot & $\begin{array}{l}\text { Ensemble des éléments qui nous définissent composé é } \\
\text { des attitudes, des croyances et des sentiments que les } \\
\text { personnes ont d'elles-mêmes. C'est un système } \\
\text { multidimensionnel défini par trois composantes: } \\
\text { cognitives, affectives et relationnelles. }\end{array}$ \\
\hline Sentiment d'auto-efficacité & 1997 & Bandura & $\begin{array}{l}\text { Croyance d'une personne en sa capacité à réaliser une } \\
\text { tâche dans une activité précise. C'est l'évaluation de } \\
\text { ses aptitudes dans des domaines particuliers qui } \\
\text { peuvent favoriser ou défavoriser l'estime de soi. }\end{array}$ \\
\hline Estime de soi & 1999 & André\&Lelord & $\begin{array}{l}\text { L'estime de soi repose sur trois ingrédients : la } \\
\text { confiance en soi, la vision de soi et l'amour de soi. Le } \\
\text { bon dosage de ces trois dimensions est indispensable } \\
\text { pour une bonne estime de soi. }\end{array}$ \\
\hline
\end{tabular}


Allès-Jardel, Metral et Scopellitti (2000) pour leur part, ont montré que les pratiques éducatives parentales ont une influence significative sur l'estime de soi et non directement sur la réussite scolaire en sixième, période propice à l'apparition de problèmes d'adaptation. Aussi la réussite scolaire est-elle influencée par le niveau d'estime de soi scolaire ainsi que par certaines variables différentielles (appartenance socioculturelle, niveau d'études de la mère et taille de la famille). Pour André (2005 : 28) : «Plus finement, il semble qu'une bonne estime de soi permette à l'enfant confronté à des difficultés d'adopter des stratégies de résolution de problèmes adaptées [...]. Tandis qu'une estime de soi déficiente est souvent associée à des stratégies inverses : repli sur soi ou réticence à parler de ses soucis, autocritique excessive ou déni des difficultés $\gg$.

Les travaux de Korman (1966) et Bhagat et Chassie (1978) ont montré que le niveau d'estime de soi est associé aux objectifs choisis, au succès remporté dans la réalisation de ces objectifs et à l'évaluation de ces objectifs. Les individus ayant une estime de soi élevée pour une tâche spécifique sont plus performants et plus satisfaits de leur programme d'études et de leur vie personnelle que les individus à faible estime de soi. Dans la perspective que l'estime de soi, influence la performance scolaire, le recours aux modèles théoriques qui s'appuient sur les composantes de l'estime de soi est indiqué pour expliquer les différences de comportements. C'est dans ce cadre que s'inscrit le modèle théorique de l'estime de soi selon André et Lelord (1999). Pour Duclos, Laporte et Ross (1995) il faut avoir un modèle impliquant l'estime de soi, la motivation, l'engagement et le sentiment d'efficacité dans le processus d'apprentissage. En effet, quand l'apprenant se concentre sur le processus d'apprentissage, corrige ses erreurs et ajuste ses stratégies, il parvient inévitablement aux succès. Il se sent alors efficace, étant conscient qu'il a adopté les bonnes attitudes et qu'il a choisi les bonnes stratégies. Plus l'apprenant réussit ce qu'il entreprend, plus il se sent efficace et fier, et plus il développe graduellement sa compétence. Il est convaincu qu'il peut relever avec succès n'importe quels défis s'il adopte les attitudes favorables et les bonnes stratégies. Le questionnaire sur l'estime de soi selon Duclos, Laporte et Ross (1995) comporte cinq (5) sous points : estime de soi sur le plan physique, estime de soi sur le plan familial, estime de soi sur le plan social, estime de soi sur le plan scolaire et estime de soi globale. Comme fréquence «toujours» correspond à 10 points, «souvent» à 7 points, «parfois» à 4 points «rarement» à 0 points. Comme résultats de 240 à 300 points équivaut à une très bonne estime de soi/estime de soi élevée; de 170 à 240 points à une assez bonne estime de soi ; de 30 à 170 points à une pauvre/faible estime de soi.

Il faut remarquer que « de piètres performances scolaires ne se répercutent pas toujours négativement sur l'estime de soi [...], de même qu'une forte estime de soi ne va pas toujours de pair avec des performances scolaires élevées » (Maintier et Alaphilippe, 2007:115).

Les auteurs Mecca, Smelser et Vasconcellos (1989) interpellent sur l'association de l'estime de soi et la réussite scolaire qui peut être influencée par d'autres facteurs, comme les capacités intellectuelles. En d'autres termes, les différences dans les capacités intellectuelles entre les élèves et non pas dans leur estime de soi peuvent provoquer des variations dans les résultats scolaires. Il faut alors donc considérer l'estime de soi comme un «sous-produit» des capacités intellectuelles des apprenants. Schunk (1991, cité par Martinot, 2001), de son côté énonce un autre déterminant qui émane de l'estime de soi qu'est la motivation. Celle-ci joue un rôle important dans la réussite scolaire des apprenants. André (2014) rappelle que l'espèce humaine est sociable et qu'un individu a besoin des autres pour sa survie physique et psychique. L'estime de soi est liée au sentiment d'appartenance et d'acceptation. Pour l'auteur, quand nous nous sentons acceptés, appréciés, voire aimés notre estime de nousmême augmente ; à contrario lorsque nous nous sentons incompris, mal aimés ou rejetés, elle baisse. Alors, notre sentiment d'estime de soi dépend de la perception que nous sommes estimés ou non par les autres (André, 2014).

Les élèves stagiaires qui participent à l'étude ont entre 14 et 18 ans. Ce sont des adolescents qui sont dans une période de leur vie très sensible et des jeunes adultes qui sont dans leur phase de transition en cherchant à se fixer par rapport au modèle de vie adopté. Les élèves stagiaires vivent une transition de l'approbation parentale vers celle des pairs qui peut déstabiliser la confiance en soi et l'estime de soi. C'est la raison pour laquelle, l'individu a besoin d'un support social, ressource importante pour renforcer l'estime de soi. Le soutien social est le réseau sur lequel l'individu peut s'appuyer lorsqu'il en a besoin. Ainsi, non seulement la disponibilité et la qualité des « relations aidantes » sont primordiales mais aussi la reconnaissance des autres. Le soutien social peut provenir de la famille, des amis, des voisins, des collègues, des organisations, etc. (Wills, 1991). Vallerand (2006 : 366) définit le soutien social comme étant des «échanges interpersonnels de ressources où une personne en aide une autre afin de lui permettre de satisfaire ses besoins ou d'atteindre des buts importants ». Les théories du soutien social mettent en évidence quatre principaux types de soutien social : le soutien émotionnel, le soutien de la compagnie, le soutien informationnel et le soutien tangible.

Le tableau 2 à la page suivante informe sur les types de soutien et la nature d'aide apportée selon certains auteurs. 


\begin{tabular}{|c|c|c|}
\hline \multicolumn{3}{|c|}{ Types de soutien et nature de l'aide apportée } \\
\hline Auteurs & Types de soutien & $\begin{array}{l}\text { Nature de l'aide } \\
\text { apportée }\end{array}$ \\
\hline \multirow[t]{4}{*}{$\begin{array}{l}\text { House (1981, } \\
\text { dans Tardy, 1985) }\end{array}$} & Émotionnel & $\begin{array}{l}\text { Manifestation de confiance, } \\
\text { d'empathie, d'amour, } \\
\text { de bienveillance }\end{array}$ \\
\hline & Instrumental & $\begin{array}{l}\text { Prêt d'argent, assistance } \\
\text { technique, transport }\end{array}$ \\
\hline & Informationnel & $\begin{array}{l}\text { Donner de l'information, } \\
\text { des avis et des conseils }\end{array}$ \\
\hline & Appréciation & $\begin{array}{l}\text { Rétroaction (exemple: tu fais } \\
\text { un bon travail) }\end{array}$ \\
\hline \multirow[t]{6}{*}{ Barrera (1981) } & Aide matérielle & $\begin{array}{l}\text { Aide financière, prêt ou don } \\
\text { d'objets physiques }\end{array}$ \\
\hline & Assistance physique & Partage des tâches \\
\hline & Interaction intime & $\begin{array}{l}\text { Expression et partage } \\
\text { des sentiments et } \\
\text { des préoccupations }\end{array}$ \\
\hline & Conseil & Avis, « guidance» \\
\hline & Rétroaction & Information sur soi-même \\
\hline & Participation sociale & Activités de loisirs \\
\hline \multirow[t]{4}{*}{ Cohen et Wills (1985) } & Estime & $\begin{array}{l}\text { Manifestation d'affection, } \\
\text { de soutien émotionnel, } \\
\text { de sympathie } \\
\text { et d'encouragement }\end{array}$ \\
\hline & Informationnel & $\begin{array}{l}\text { Aide dans la définition } \\
\text { et la compréhension } \\
\text { d'événements probléma- } \\
\text { tiques; éducation, conseils, } \\
\text { références }\end{array}$ \\
\hline & Accompagnement social & $\begin{array}{l}\text { Activités de loisirs } \\
\text { et activités sociales }\end{array}$ \\
\hline & Instrumental & Aide financière et matérielle \\
\hline
\end{tabular}

Source: Beauregard et Dumont, 1996

Le soutien émotionnel est l'apport d'inquiétude, d'empathie, d'affection, d'amour, etc (Slevin, Nichols, Downer, Wilson, Lister, Arnott, Maher, Souhami, Tobias, Goldstone \& Cody, 1996; Langford, Bowsher, Moloney \& Lillis, 1997) et il permet aux individus de savoir qu'ils sont valorisés et importants (Slevin, Nichols, Downer, Wilson, Lister, Arnott, Maher, Souhami, Tobias, Goldstone \& Cody, 1996). Le soutien de la compagnie donne à une personne un sentiment d'appartenance (Wills 1991) et d'acceptation. La recension des écrits scientifiques indique que le soutien social exerce une influence positive sur l'estime de soi, la gestion du stress et sur la santé (Beaumeister \& Vohs, 2007). Vaux (1992) stipule que la source ou la provenance du soutien social est d'une importance capitale, notamment lorsqu'il est question de sa mesure. Dans la même perspective, Streeter et Franklin (1992) jugent essentiel de faire la distinction entre les sources formelles et les sources informelles. Les sources informelles sont le soutien des amis, de la famille, etc., tandis que les sources formelles proviennent des organisations formelles (services gouvernementaux, agences privées). Pour notre étude, les sources informelles et formelles (soutien de la famille, des pairs, du personnel d'encadrement) associées à l'environnement social de l'élève sont retenus. Pour Vaux (1988: 28) «le réseau de soutien est un sous-ensemble du réseau social, vers qui la personne se tourne (ou pourrait se tourner) pour obtenir de l'aide... ». Lorsqu'on parle des ressources du réseau de soutien, on fait référence à la grandeur du réseau de soutien, à sa structure ou encore à ses caractéristiques relationnelles (Vaux, Phillips, Holly, Thomson, Williams et Stewart, 1986). Les instruments de mesure des ressources du réseau sont l'identification des rapports (indicateurs des ressources sociales susceptibles d'offrir du soutien, lors d'une crise) reliant la personne à sa famille, à ses amis, à ses pairs (Streeter et Franklin, 1992).

La théorie de référence de cette étude est celle de l'estime de soi d'André et Lelord (1999) qui stipule que l'estime de soi repose sur trois piliers qui sont l'amour de soi, la vision de soi et la confiance en soi. L'amour de soi conduit l'individu à s'aimer malgré ses défauts, ses limites et les échecs rencontrés. Il n'empêche, ni la souffrance, ni le doute en cas de difficultés. Il permet de se reconstruire après un échec 
et protège l'individu du désespoir. En tant que la vision de soi, l'estime est définie comme le regard que l'on porte sur soi. C'est aussi l'évaluation, fondée ou non, que l'on fait de ses qualités et de ses défauts. L'important c'est d'avoir la conviction que l'on a d'être porteur de qualités ou de défauts, de potentialités voire de limitations. La confiance en soi quant à elle, s'applique à nos actes. Être confiant, c'est penser que l'on est capable d'agir de manière adéquate dans les situations importantes. Ces trois piliers de l'estime de soi entretiennent généralement des liens d'interdépendance : l'amour de soi (s'aimer) permet une vision positive de soi (croire en ses capacités) influençant positivement la confiance en soi (agir sans craindre de façon excessive l'échec). Ces trois piliers conduisent à une estime de soi élevée. Pour une estime de soi élevée, il faut inévitablement un équilibre entre les trois (3) composantes. Dans ce contexte, l'individu utilise plus efficacement les stratégies et les compétences qu'il a développées. Il persévère davantage dans le travail scolaire malgré les difficultés rencontrées. A contrario l'individu ayant une estime de soi est faible pense qu'il est incapable de réussir ses tâches scolaires, manque de persévérance et a tendance à abandonner devant les difficultés. Cependant de piètres performances scolaires ne se répercutent pas toujours négativement sur l'estime de soi (Crocker et Park, 2004 ; Mecca, Smelser et Vasconcellos, 1989), de même qu'une forte estime de soi ne conduit pas toujours à des performances scolaires élevées (Marsh, 1990).

L'objectif de cette étude est d'analyser la relation entre l'estime de soi, le soutien social et la performance scolaire des élèves. L'hypothèse stipule que l'estime de soi élevée, le soutien social conduit à une bonne performance scolaire des élèves.

\section{MÉTHODOLOGIE}

\section{Terrain et population de l'étude}

Le terrain de recherche est le Lycée Professionnel Hôtelier de Cocody. L'étude a été effectuée auprès des élèves stagiaires du Lycée hôtelier de Cocody, avec un échantillon de disponibilité et de convenance composé de cinquante-cinq (55) élèves stagiaires dont vingt-neuf (29) filles et vingt-six (26) garçons.

\section{Techniques de recueil et d'analyse des données}

Il faut signaler que cette étude a été précédée par une pré-enquête sur l'estime de soi, le soutien social et la performance scolaire des élèves stagiaires.
Les techniques de recueil des données utilisées au cours de cette étude proprement dite sont la recherche documentaire, le questionnaire et l'entretien semi-directif. La recherche documentaire utilisée dans cette étude a permis de recueillir des données factuelles et existantes pour atteindre l'objectif visé en comparant les bulletins de notes des enquêtés du premier et du deuxième trimestre; en renforçant les connaissances sur le sujet de recherche pour bien mener l'entretien à travers un guide d'entretien. Le questionnaire sur l'estime de soi selon Duclos, Laporte et Ross (1995) a été administré aux élèves stagiaires. Le questionnaire permet en un temps record la récolte de nombreuses données. L'administration de ce questionnaire a permis d'obtenir des scores d'estime de soi des enquêtés de notre échantillon et de les classer selon leur estime de soi (estime de soi élevée, estime de soi faible). Les performances scolaires représentent la moyenne obtenue par chaque élève au deuxième trimestre. Lorsque l'enquêté a une moyenne supérieure ou égale à dix (10) $[\geq 10]$ sur 20 , il a une bonne performance scolaire ; par contre s'il a une moyenne inférieure à dix (10) $[<10]$ sur 20, il a une mauvaise performance et est en situation d'échec scolaire. Les données recueillies ont fait l'objet d'un traitement statistique avec le logiciel SPSS. La nécessité d'étudier le lien entre la fréquence du soutien social et la performance scolaire ainsi que la relation entre l'estime de soi et la performance scolaire nous amène à comparer le groupe qui a toujours bénéficié du soutien social au groupe qui en a reçu souvent et quelques fois ainsi que le groupe à estime de soi élevée/très bonne estime de soi au groupe à estime de soi faible. Ces deux groupes étant indépendants, nous avons appliqué le test statistique Khi carré. A travers cette procédure, nous avons obtenu des données chiffrées qui ont conduit à l'élaboration des tableaux de pourcentage. Ceci a amené à faire ressortir lien entre le soutien social et la bonne performance scolaire ainsi que la relation entre l'estime de soi élevée et la bonne performance scolaire. Avec le guide d'entretien, les réponses des enquêtés ont conduit à des résultats variés sur le contenu de leur soutien social leur permettant d'avoir de bonnes performances scolaires; en somme les répercussions positives du soutien social sur leurs performances scolaires. Les données recueillies ont fait l'objet d'une analyse qualitative.

\section{RÉSULTATS ET DISCUSSION}

1. Sexe des enquêtés

Le sexe des apprenants est précisé dans le tableau ci-dessous.

\begin{tabular}{|l|l|l|l|l|l|}
\hline \multicolumn{2}{|c|}{} & Fréquence & Pourcentage & $\begin{array}{l}\text { Pourcentage } \\
\text { valide }\end{array}$ & $\begin{array}{l}\text { Pourcentage } \\
\text { cumulé }\end{array}$ \\
\hline \multirow{3}{*}{ Valide } & Garçon & 26 & 47,3 & 47,3 & 47,3 \\
\cline { 2 - 6 } & Fille & 29 & 52,7 & 52,7 & 100,0 \\
\cline { 2 - 6 } & Total & 55 & 100,0 & 100,0 & \\
\hline
\end{tabular}

Source: Enquête sur le terrain 
Il apparait que l'échantillon d'étude est composé majoritairement de filles $(52,7 \%)$. Les élèves de sexe masculin représentent quant à eux $47,3 \%$ des enquêtés.

\section{2. Étude du lien entre le niveau d'estime de soi et le niveau de performance scolaire}

Il est également postulé que le niveau de performance scolaire de l'élève varie selon son niveau d'estime de soi. Dans ce sens, le tableau croisé cidessous a été réalisé.

\begin{tabular}{|c|c|c|c|c|}
\hline \multicolumn{5}{|l|}{ Tableau croisé } \\
\hline & & Niveau d'Estime de $\mathrm{S}$ & & \\
\hline & & Estime de Soi élevée & Estime de Soi faible & Total \\
\hline \multirow{4}{*}{ Niveau de Performance scolaire } & \multirow[b]{2}{*}{ Bonne Performance } & 32 & 14 & 46 \\
\hline & & $69,6 \%$ & $30,4 \%$ & $100,0 \%$ \\
\hline & \multirow{2}{*}{ Mauvaise Performance } & 3 & 6 & 9 \\
\hline & & $33,3 \%$ & $66,7 \%$ & $100,0 \%$ \\
\hline \multirow{2}{*}{ Total } & & 35 & 20 & 55 \\
\hline & & $63,6 \%$ & $36,4 \%$ & $100,0 \%$ \\
\hline
\end{tabular}

Source: Enquête sur le terrain

La lecture du tableau croisé ci-dessus indique que deux tiers (2/3) des élèves ayant une bonne performance scolaire, soit $69,6 \%$, ont une estime de soi élevée. A l'opposé, les élèves qui ont une mauvaise performance scolaire ont dans leur majorité $(66,7 \%)$, une faible estime de leur personne. Ce contraste au niveau de l'estime de soi entre les élèves performants et les élèves non-performants nous amène à supposer l'existence d'une relation entre l'estime de soi et la performance scolaire de l'élève. Dans le but de tester cette relation, il est nécessaire de recourir au test statistique du Khi-deux.

\begin{tabular}{|l|l|l|l|l|l|}
\hline Tests du khi-carré & Valeur & ddl & $\begin{array}{l}\text { Signification asymptotique } \\
\text { (bilatérale) }\end{array}$ & $\begin{array}{l}\text { Sig. exacte } \\
\text { (bilatérale) }\end{array}$ & $\begin{array}{l}\text { Sig. exacte } \\
\text { (unilatérale) }\end{array}$ \\
\hline khi-carré de Pearson $^{\text {Correction pour continuité }}{ }^{\mathrm{b}}$ & $4,270^{\mathrm{a}}$ & 1 &, 039 & & \\
\hline Rapport de vraisemblance & 2,848 & 1 &, 091 & & \\
\hline Test exact de Fisher & 4,111 & 1 &, 043 & &, 059 \\
\hline Association linéaire par linéaire & 4,193 & 1 &, 041 & &, 048 \\
\hline N d'observations valides & 55 & & & & \\
\hline a. 1 cellules (25,0\%) ont un effectif théorique inférieur à 5. L'effectif théorique minimum est de 3,27. \\
\hline b. Calculée uniquement pour une table 2x2 \\
\hline
\end{tabular}

\begin{tabular}{|l|l|l|l|}
\hline \multicolumn{2}{|l|}{ Mesures symétriques } & Valeur & $\begin{array}{l}\text { Signification } \\
\text { approximative }\end{array}$ \\
\hline \multirow{2}{*}{ Nominal par Nominal } & Phi &, 279 &, 039 \\
\cline { 2 - 4 } & V de Cramer &, 279 &, 039 \\
\cline { 2 - 4 } & Coefficient de contingence &, 268 &, 039 \\
\hline N d'observations valides & 55 & \\
\hline
\end{tabular}

L'hypothèse nulle $\mathrm{H} 0$ stipule qu'il n'existe pas de lien entre ces deux variables «estime de soi » et «performance scolaire ». Vérifions H0 au seuil de 5\%. Les deux variables en présence étant qualitatives, le test statistique approprié est le test khi deux d'indépendance. Les résultats indiquent que le test du Khi-deux n'est pas valide car $25 \%$ des cellules ont un effectif théorique inférieur à 5. Dans ce sens, il est fait recours au test du rapport de vraisemblance qui indique une $p$-value $(p=0,043)$ inférieure à 0,05 . Le test du rapport de vraisemblance confirme donc la significativité de la relation et permet d'affirmer au seuil de risque $\alpha=5 \%$ que la performance scolaire de l'élève est significativement liée à son estime de soi. En d'autres termes, l'élève aura probablement une bonne performance à l'école s'il dispose d'une estime de soi élevée. Mais si son estime de soi se révèle faible, il aura tendance à enregistrer une mauvaise performance scolaire. Notons que la relation entre ces deux variables est faible car le V de Cramer = 0,279.

Dans les lignes suivantes nous insérons certains verbatims des enquêtés ayant une estime de soi élevée et une bonne performance scolaire:

«Moi, j'ai toujours reçu un soutien régulier de la part de ma famille, j'écoute toujours les bons conseils venant de ma famille et de mes amis, $j$ 'ai confiance en 
moi et je sais que je peux réussir parce que j'en ai les capacités.,.» (Enquêté TiS).

«Je sais que je suis capable de réussir aux évaluations, la raison pour laquelle j'étudie... comme je ne veux pas de surprise, je prends conseille auprès de mes amis et de mes enseignants...ma famille m'accorde beaucoup d'attention....» (Enquêté RoL).

«Je me connais bien et j'ai toujours répondu à mes besoins et je sais aussi que je peux toujours trouver la solution aux difficultés. Je prends mes précautions pour mieux étudier et je sais... » (Enquêté CaL).

«Pour moi, il faut avoir une vision claire de qui on est...étant élève je dois toujours étudier pour réussir... et aussi j'ai confiance en moi...» (Enquêté $\mathrm{ToS})$.
«Pour moi, il faut avoir une vision claire de qui on est...étant élève je dois toujours étudier pour réussir... et aussi j'ai confiance en moi...» (Enquêté TaL).

«Je suis toujours fière de moi et je suis sûre de moi pendant les évaluations parce que j'étudie au jour le jour...» (Enquêté ReL).

«Je me connais et je sais que je dois réussir, c'est la raison pour laquelle je suis avec les amis qui aiment aussi le travail et qui ont une vision claire de la vie. Je ne peux que réussir...»(Enquêté ZiZ)

\section{Forme de soutien social reçu}

Interrogés sur la forme du soutien reçu, les élèves ont fourni les réponses consignées dans le tableau ci-dessous.

\begin{tabular}{|l|l|l|l|l|l|}
\hline \multicolumn{2}{|c|}{} & Fréquence & Pourcentage & $\begin{array}{l}\text { Pourcentage } \\
\text { valide }\end{array}$ & $\begin{array}{l}\text { Pourcentage } \\
\text { cumulé }\end{array}$ \\
\hline \multirow{3}{*}{ Valide } & Instrumentale & 9 & 16,4 & 16,4 & 16,4 \\
\cline { 2 - 6 } & Informative & 19 & 34,5 & 34,5 & 50,9 \\
\cline { 2 - 6 } & Emotionnelle & 27 & 49,1 & 49,1 & 100,0 \\
\cline { 2 - 6 } & Total & 55 & 100,0 & 100,0 & \\
\hline
\end{tabular}

Source: Enquête sur le terrain

Le soutien reçu par les élèves se présente majoritairement $(49,1 \%)$ sous la forme d'un soutien émotionnel (famille, pairs). Plus d'un tiers des élèves $(34,5 \%)$ ont admis recevoir un soutien informatif (enseignant). Enfin, moins d'un élève sur cinq $(16,4 \%)$ reçois un soutien sous forme instrumentale.

Ce soutien reçu a été investigué sous sa fréquence, car il est postulé dans cette étude que le fait de bénéficier régulièrement d'un soutien social (instrumental, informatif, émotionnel) pourrait influencer les performances scolaires de l'élève.

Les lignes suivantes liste certains verbatims des enquêtés au niveau du soutien émotionnel, instrumental et informatif:

\section{Soutien émotionnel}

«Ma famille c'est ma vie, ...elle est là pour moi, elle est bienveillante et je me sens bien sdans toutes mes décisions, car je sais que je peux compter sur le soutien de ma famille...» (Enquêté SoL)

«Je reçois beaucoup d'amour de la part de ma famille, elle m'encourage dans tout ce que j'entreprends et tout me réussit...mes amis m'apprécie et me soutiennent beaucoup » (Enquêté $\mathrm{QoH})$

\section{Soutien instrumental}

«Pour moi, je reçois de l'argent pour mes besoins en me procurant tout ce qui est indispensable pour ma vie...» (Enquêté ToS).
«Je n'ai aucun souci technique pour la bonne marche de mes études,... j'ai tout le matériel dont j'ai besoin.» (Enquêté $\mathrm{ZiS}$ ).

"Que de bienfaits,... je suis à l'abri de tous besoins, je ne manque de rien.» (Enquêté $\mathrm{BeM}$ )

«Tout ce qui doit être acheté pour les études est disponible, $\mathrm{j}$ 'ai tout... » (Enquêté RiL)

\section{Soutien informatif}

«Mes enseignants sont toujours disponibles pour moi, je m'adresse à eux pendant les pauses, ... à la fin de cours pour les conseils.» (Enquêté TiS).

"...Pour avoir de bons résultats, il faut toujours avoir accès aux bonnes informations, aux exercices pratiques et réels qui conduisent à la réussite...» (Enquêté HaS).

«Les informations actuelles sur la correction des exercices sont faciles d'accès auprès des enseignants,... j'ai toujours eu recours à toutes ces informations avant les évaluations...» (Enquêté QiT).

«...Toutes les informations sont disponibles, moi je vais toujours à l'information pour avoir de bons résultats...» (Enquêté QoL). 
4. Étude du lien entre la fréquence du soutien social et le niveau de performance scolaire
Dans le but de vérifier le lien entre la fréquence du soutien social et le niveau de performance scolaire, le tableau croisé ci-dessous a été élaboré.

\begin{tabular}{|c|c|c|c|c|c|}
\hline \multicolumn{6}{|l|}{ Tableau croisé } \\
\hline & & \multicolumn{3}{|c|}{$\begin{array}{l}\text { En moyenne, à quelle fréquence avez-vous reçu du } \\
\text { soutien social? }\end{array}$} & \multirow[t]{2}{*}{ Total } \\
\hline & & Toujours & Souvent & Quelques fois & \\
\hline \multirow{4}{*}{$\begin{array}{l}\text { Niveau de Performance } \\
\text { scolaire }\end{array}$} & \multirow{2}{*}{$\begin{array}{l}\text { Bonne } \\
\text { Performance }\end{array}$} & 36 & 9 & 1 & 46 \\
\hline & & $78,3 \%$ & $19,6 \%$ & $2,2 \%$ & $100,0 \%$ \\
\hline & \multirow{2}{*}{$\begin{array}{l}\text { Mauvaise } \\
\text { Performance }\end{array}$} & 5 & 1 & 3 & 9 \\
\hline & & $55,6 \%$ & $11,1 \%$ & $33,3 \%$ & $100,0 \%$ \\
\hline \multirow[t]{2}{*}{ Total } & & 41 & 10 & 4 & 55 \\
\hline & & $74,5 \%$ & $18,2 \%$ & $7,3 \%$ & $100,0 \%$ \\
\hline
\end{tabular}

Source: Enquête sur le terrain

Les données obtenues indiquent que chez la majorité des élèves qui ont une bonne performance scolaire, la majorité $(78,3 \%)$ déclare recevoir «toujours » un soutien social. Ils représentent 19,6\% à recevoir «souvent» et $2,2 \%$ à recevoir «quelques fois » un soutien social. A l'opposé, un tiers $(33,3 \%)$ des élèves qui ont de mauvaises performances à l'école disposent «quelques fois» d'un soutien social.
Seulement, un peu plus de la moitié des élèves qui ont de mauvaises performances à l'école $(55,6 \%)$ reçoivent «toujours » un soutien social. Ces données semblent suggérer un lien entre la performance scolaire de l'élève et la fréquence du soutien reçu. Dans ce cadre, il est fait recours au test de Khi-deux afin de vérifier la significativité de la relation.

\begin{tabular}{|l|l|l|l|}
\hline Tests du khi-carré & Valeur & ddl & $\begin{array}{l}\text { Signification asymptotique } \\
\text { (bilatérale) }\end{array}$ \\
\hline & $10,865^{\mathrm{a}}$ & 2 &, 004 \\
\hline khi-carré de Pearson & 7,616 & 2 &, 022 \\
\hline Rapport de vraisemblance & 5,865 & 1 &, 015 \\
\hline Association linéaire par linéaire & 55 & & \\
\hline N d'observations valides & $\begin{array}{l}\text { a. 3 cellules (50,0\%) ont un effectif théorique inférieur à 5. L'effectif théorique minimum } \\
\text { est de ,65. }\end{array}$ \\
\hline
\end{tabular}

\begin{tabular}{|l|l|l|l|}
\hline \multicolumn{2}{|l|}{ Mesures symétriques } & Valeur & Signification approximative \\
\hline \multirow{3}{*}{ Nominal par Nominal } & Phi &, 444 &, 004 \\
\cline { 2 - 4 } & V de Cramer &, 444 &, 004 \\
\cline { 2 - 4 } & Coefficient de contingence &, 406 &, 004 \\
\hline N d'observations valides & 55 & \\
\hline
\end{tabular}

L'hypothèse nulle H0 stipule qu'il n'existe pas de lien entre ces deux variables « fréquence de soutien social » et «performance scolaire ». Vérifions H0 au seuil de $5 \%$. Les deux variables en présence étant qualitatives, le test statistique approprié est le test khi deux d'indépendance. Les résultats indiquent que le test du Khi-deux n'est pas valide car 50\% des cellules ont un effectif théorique inférieur à 5 . Dans ce sens, il est fait recours au test du rapport de vraisemblance qui indique une $p$-value $(p=0,022)$ inférieure à 0,05 . Le test du rapport de vraisemblance confirme donc la significativité de la relation et permet d'affirmer au seuil de risque $\alpha=5 \%$ que le fait pour les élèves de recevoir régulièrement du soutien social de la part de leur famille, améliore significativement leur performance scolaire. En d'autres termes, le soutien social aux élèves est un facteur conditionnant les résultats scolaires de ces derniers. Notons que la relation entre ces deux variables est modérée car le $\mathrm{V}$ de Cramer $=0,444$.

Les lignes suivantes nous répertorions certains verbatims des enquêtés ayant obtenus du soutien social de la part de la famille et faisant partis de ceux qui ont une bonne performance scolaire:

«Moi, j'ai toujours reçu un soutien régulier de la part de ma famille et surtout de mes parents qui sont toujours là pour moi, ils ont confiance en moi, ils m'écoutent et me donnent des conseils.» (Enquêté TiS).

«Je suis fière de ma famille, parce qu'elle me soutient réellement et régulièrement et toujours, peu importe dans quelle difficulté je me trouve, elle est pour moi un soutien infaillible. » (Enquêté PaL). 
«Ma famille est toujours à ma disposition, elle m'accorde beaucoup d'attention...je me tourne toujours vers elle en cas de besoin...elle est là pour moi.» (Enquêté RoL).

«Ma réussite, c'est ma famille. Elle est mon soutien. Je ne sais pas ce que je vais devenir à l'école si ma famille n'est pas toujours là pour moi...» (Enquêté $\mathrm{CaL})$.

«Ma famille me soutient régulièrement, tout va bien au lycée, je n'ai pas de problèmes avec mes résultats scolaires» (Enquêté TeL).

\section{DISCUSSION}

L'analyse des résultats obtenus permet de confirmer l'hypothèse selon laquelle l'estime de soi élevée, le soutien social conduit à une bonne performance scolaire des élèves. En d'autres termes, les élèves stagiaires ayant une estime de soi élevée et bénéficiant d'un soutien social ont une bonne performance scolaire.

Les résultats de cette étude ont permis, au seuil de confiance de $5 \%$, de conclure en l'influence significative de l'estime de soi des élèves stagiaires sur leurs performances scolaires. Concrètement, les apprenants ayant une estime de soi élevée achèvent, le plus souvent, leurs travaux dans le temps imparti. Cependant, une estime de soi élevée ne conduit pas obligatoirement à de bonnes performances scolaires et ceci vice versa (Maintier et Alaphilippe, 2007). C'est ainsi que Mecca, Smelser et Vasconcellos (1989) nous interpellent sur l'influence d'autres facteurs sur l'estime de soi et la réussite scolaire. En outre, non seulement la motivation détermine le niveau de l'estime de soi Schunk (1991, cité par Martinot, 2001) mais l'estime de soi est liée au sentiment d'appartenance et d'acceptation (André, 2014). L'analyse qualitative, à travers l'entretien semi directif, indique que les élèves stagiaires ayant une estime de soi élevée s'approchent de leurs enseignants et leurs pairs pour pallier leurs difficultés scolaires; ceci leur permet de mieux comprendre les leçons et ainsi améliorer leur rendement scolaire; contrairement à leurs pairs ayant un niveau d'estime de soi faible, qui lorsque leur méthode s'avère inefficace, abandonnent la tâche. Or, un travail inachevé ne peut garantir une performance chez l'apprenant. La supériorité des apprenants qui ont un niveau d'estime de soi élevé par rapport à leurs pairs de niveau d'estime de soi faible semble provenir $\mathrm{du}$ fait qu'ils sont persévérants, utilisent des méthodes de travail adéquates et obtiennent régulièrement des encouragements de la part de leur famille. Ceci va dans le même sens que les recherches d'Allès-Jardel, Metral et Scopellitti (2000) qui ont mis en exergue l'influence significative des pratiques éducatives parentales sur l'estime de soi. A l'école, l'élève n'est motivé à apprendre, à réussir que s'il a la conviction d'être capable de réaliser ce qu'on attend de lui au cours de l'acte pédagogique. Il développe alors une image positive ou négative de lui-même en tant que sujet apprenant. Cette représentation de soi a une influence sur son engagement et ses résultats (Bawa, 2002).

Cette étude a mis par ailleurs, en exergue le rôle significatif du soutien social reçu par l'apprenant comme facteur conditionnant ses performances scolaires. Wills (1991) nous éclaire sur la provenance du soutien social (entre autres la famille, les pairs ou les enseignants). Ainsi cette étude a souligné ces trois dimensions du soutien social relatives à l'émotionnel, à l'informatif et à l'instrumental. Ces dimensions se retrouvent dans la définition de Vallerand (2006: 366) qui perçoit le soutien social comme des « échanges interpersonnels de ressources où une personne en aide une autre afin de lui permettre de satisfaire ses besoins ou d'atteindre des buts importants ». La fréquence du soutien social aux élèves est un facteur conditionnant les résultats scolaires de ces derniers. Autrement dit, la fréquence du soutien social conduit inévitablement à une bonne performance scolaire.

\section{CONCLUSION}

L'objectif cette étude est d'analyser le lien entre l'estime de soi, le soutien social et la performance scolaire des élèves. Elle met en exergue le niveau d'estime de soi, le soutien social et observe en quoi ces deux (2) variables influencent la performance scolaire des élèves stagiaires. L'hypothèse met l'accent sur l'existence de la relation entre l'estime de soi élevée, le soutien social et la performance scolaire. Les résultats montrent que les élèves stagiaires ayant une estime de soi élevée, recevant du soutien social ont une bonne performance scolaire. En outre, à travers les entretiens notre préenquête, il ressort que le soutien social régulier (émotionnel, informatif et instrumental) des apprenants de la part des agents sociaux et scolaires (famille, pairs, enseignants) contribue à une estime de soi élevée surtout lorsque ceux-ci rencontrent des difficultés durant leur parcours scolaire. Les parents fournissant un attachement sécurisant et un cadre nécessaire pour le bien-être de l'apprenant, lui permettant d'avoir une estime de soi élevée. Cette étude est cependant limitée dans le sens où elle ne tient pas compte des facteurs financiers et sociodémographiques (Meuret et al. Morlaix, 2006; Forquin, Bourdieu et al. Passeron, 1971; Scheerens, 2000; Kouakou et al. Kablan, 2015).

\section{RÉFÉRENCES}

- $\quad$ Alles-Jardel, M., Metral, V. Et Scopellitti, S. (2000). Pratiques éducatives parentales, estime de soi et réussite scolaire d'élèves de sixième. In: La revue internationale de l'éducation familiale, 4(1), (63-91).

- André, C. et Lelord, F. (1999). L'Estime de soi : S'aimer pour mieux vivre avec les autres. Paris : Odile Jacob.

- Baudelot, C.H. et Establet, R. (1979). L'école primaire divise. Paris: Petite collection Maspero.

- Bariaud, F., Bourcet, C. (1998). L'estime de soi à 
l'adolescence. In: BOLOGNINI, M., Preteur, Y. (dir.). Estime de soi, perspectives développementales. Lausanne, Delachaux et Niestlé.

- Bawa, I. H. (2007). Estime de soi et performances scolaires chez des adolescents (TOGO). Mémoire de DESS dans la Filière de Psychologie Appliquée à l'Université de Lomé. Lomé : Université de Lomé.

- Beaumeister, R. F., \& Vohs, K. D. (dir.) (2007). Encyclopedia of Social Psychology. Thousand Oaks, CA: Sage Publications.

- $\quad$ Beauregard, L.A., Bouffard, R. et Duclos, G. (2000). Estime de soi et compétences sociales chez les 8 à 12 ans. Hôpital Sainte-Justine. Montréal : Editions du CHU Sainte-Justine.

- Beauregard, L. et Dumont, S. (1996). La mesure du soutien social. In : Service social, 45 (3), (55-76).

- Bhagat, R. S. et Chassie, M. B. (1978). The role of self-esteem and locus of control in the differential prediction of performance, program satisfaction, and life satisfaction in an educational organization. In: Journal of Vocational Behavior, 13 (3), (317-326).

- Bloom, B. S. (1979). Caractéristiques individuelles et apprentissages scolaires (V. De Lansheere, Trad.). Paris: Fernand Nathan. (Oeuvre originale publiée en 1966).

- Bourdieu, P. et Passeron, J.-CL. (1970). La reproduction. Eléments pour une théorie du système d'enseignement. Paris : Les Editions de Minuit.

- Coopersmith, S. (1967). The antecedents of selfesteem. San Francisco, CA, Freeman.

- Crocker, J., \& PARK, L. E. (2004). The Costly Pursuit of Self-Esteem. In: Psychological Bulletin, 130 (3), (392-414).

- Duru-Bellat, M. (2002). Les inégalités sociales à l'école. Paris : Presses Universitaires de France.

- Famose, J.P., Guerin, F. et Sarrazin, P. (2005). Les croyances sur soi : clarification conceptuelle, formation et relation à la performance sportive. In : O. Rascle et P. SARRAZIN (Eds.) Croyances et performance sportive. Processus sociocognitifs associés aux comportements sportifs (19-52). Paris: Editions Revue EPS.

- Forquin, J.C.L., Bourdieu, P. et Passeron, J.C.L. (1971). La reproduction. Eléments pour une théorie du système d'enseignement. In: Revue française de pédagogie, 15, (39-44).

- Gerardi, S. (1990). Academic self-concept as a predictor of academic success among minority and low-socio-economic status students. In: Journal of College Student Development, 31, (402-407).

- Joly, CH. (2011). Connais-toi toi-même. La connaissance de soi éclaire tout homme sur ce qu'il est et ce qu'il peut... Socrate Ou Quelles stratégies pédagogiques mettre en place pour permettre à l'élève de SEGPA de restaurer son estime de soi et de renouer avec la réussite scolaire? Mémoire professionnel à l'Académie de Nantes. Année universitaire 2010 - 2011, CAPA-SH Option F. Nantes : Académie de Nantes.

- Kouakou, N. et Kablan, F. B. (2015). Analyse des déterminants de la performance scolaire des établissements du secondaire public au Baccalauréat, session 2015 : cas de la Côte d'Ivoire. In : Revue Universitaire des Sciences de l'Éducation, 5(44 - 60).

- Langford, C. P. H., Bowsher, J., Maloney, J. P., \& Lillis, P. P. (1997). Social support: a conceptual analysis. Journal of advanced nursing, 25(1), 95-100.

- Marsh, H. W. (1990). The structure of academic selfconcept: The Marsh/Shavelson model. In:, Journal of Educational Psychology, 82(4), (623-636).

- Mecca, A., Smelser, N. J., \& Vasconcellos, J. (Eds.). (1989). The social importance of self-esteem. Univ of California Press.

- Meuret, D. (2000). Établissements scolaires: ce qui fait la différence. In : l'Année sociologique, 50 (2), $(545-555)$.

- Meuret, D., \& Morlaix, S. (2006). L'influence de l'origine sociale sur les performances scolaires: par où passe-t-elle? Revue française de sociologie, 47(1), 49-79.

- $\quad$ Pierrehumbert, B., Zanone, F., Kauer-Tchicaloff, C., \& Plancherel, B. (1987). Image de soi et échec scolaire. Bulletin de psychologie.

- Rocheleau, J. (1998). Une approche de gestion pour l'école informatisée. Montréal : Laboratoire en informatique cognitive et environnements de formation (LICEF).

- Scheerens, J. (2000). Improving school effectiveness. In: Fundamentals of Educational Planning, (68). Paris: UNESCO International Institute for Educational Planning.

- Slevin, M. L., Nichols, S. E., Downer, S. M., Wilson, P., Lister, T. A., Arnott, S., ... \& Cody, M. (1996). Emotional support for cancer patients: what do patients really want?. British journal of cancer, 74(8), 1275-1279.

- $\quad$ Streeter, C. L., \& Franklin, C. (1992). Defining and measuring social support: Guidelines for social work practitioners. Research on Social Work Practice, 2(1), 81-98.

- Vallerand, R. J. (2006). Les fondements de la psychologie sociale. 2ème édition. Québec: Gaetan Morin.

- Vaux, A. (1988). Social Support. Theory, Research, and Intervention. New York: Praeger.

- Vaux, A., Phillips, J., Holly, L., Thomson, B., Williams, D., \& Stewart, D. (1986). The social support appraisals (SS-A) scale: Studies of reliability and validity. American Journal of Community Psychology, 14(2), 195-218.

- Wills, T. A. (1991). Social support and interpersonal relationships.

Cite This Article: Essiomle Yawa Ossi (2022). Estime De Soi, Soutien Social Et Performance Scolaire Des Élèves Stagiaires Du Lycée Hôtelier De Cocody (Côte D'ivoire). East African Scholars J Edu Humanit Lit, 5(1), 1-10. 